Article

\title{
Self-Q-Switch and CW Operation of a Tunable Dual-Wavelength Er/Yb Double-Clad Fiber Laser
}

\author{
Baldemar Ibarra-Escamilla ${ }^{1}$, Manuel Durán-Sánchez ${ }^{2, *}$, Berenice Posada-Ramírez ${ }^{1}$, \\ Patricia Prieto-Cortés ${ }^{3}$, Ricardo I. Álvarez-Tamayo ${ }^{4}$ and Evgeny A. Kuzin ${ }^{1}$ \\ 1 Optics Department, Instituto Nacional de Astrofísica, Óptica y Electrónica, L. E. Erro 1, \\ Sta. Ma. Tonantzintla, Puebla 72824, Mexico; baldemar@inaoep.mx (B.I.-E.); b.posada@inaoep.mx (B.P.-R.); \\ ekuz@inaoep.mx (E.A.K.) \\ 2 Consejo Nacional de Ciencia y Tecnología (CONACYT)—Optics Department, \\ Instituto Nacional de Astrofísica, Óptica y Electrónica, L. E. Erro 1, Sta. Ma. Tonantzintla, \\ Puebla 72824, Mexico \\ 3 Facultad de Ciencias Físico Matemáticas, Universidad Autónoma de Nuevo León, Av. Universidad S/N, \\ Ciudad Universitaria, San Nicolás de los Garza, Nuevo León 66455, Mexico; pattyprieto@hotmail.com \\ 4 Consejo Nacional de Ciencia y Tecnología (CONACYT)_Facultad de Ciencias Físico Matemáticas, \\ Universidad Autónoma de Nuevo León, Av. Universidad S/N, Ciudad Universitaria, \\ San Nicolás de los Garza, Nuevo Leon 66455, Mexico; rialvarez@conacyt.mx \\ * Correspondence: mduransa@conacyt.mx; Tel.: +52-222-266-3100 (ext. 8112)
}

Received: 15 December 2017; Accepted: 22 January 2018; Published: 25 January 2018

Featured Application: High-power light sources, terahertz generation, thulium-doped fiber laser, pump power sources.

\begin{abstract}
In this paper, a double-clad Er/Yb fiber laser with self-Q-switched and continuous wave operation depending on the pump power range is experimentally demonstrated. The linear cavity is formed on one side by a pair of cascaded tunable fiber Bragg gratings used for the selection and tuning of the generated laser lines. On the opposite side, a fiber optical loop mirror with high birefringence fiber in the loop is used to adjust the intra-cavity losses to obtain dual-wavelength emission by temperature changes on the fiber loop. Continuous wave dual-wavelength laser operation is obtained for tunable separation of the generated laser lines in a range from 1 to $7 \mathrm{~nm}$, maximum output power of $3.6 \mathrm{~W}$ with a pump power of $10 \mathrm{~W}$ and laser wavelengths linewidth of $\sim 0.2 \mathrm{~nm}$. Self-Q-switched laser pulses are obtained with low pump power in a range from 322 to $890 \mathrm{~mW}$. Q-switched pulses with minimum pulse duration of $\sim 1.5 \mu$ s and maximum pulse energy of $\sim 3.5 \mu \mathrm{J}$ are obtained.
\end{abstract}

Keywords: fiber lasers; Q-switched lasers; fiber Bragg gratings; $\mathrm{Er} / \mathrm{Yb}$ double-clad fibers; fiber optical loop mirror

\section{Introduction}

Fiber lasers have been of increasing interest because of their reliability, low-cost and performance efficiency. In the $1.55 \mu \mathrm{m}$ wavelength range, fiber lasers have been extensively studied due to their advantages of relatively "eye safe" operation and good free-space and fiber guided transmission with low losses. These advantages make them attractive to develop high power fiber lasers for applications in industrial manufacturing, medical surgery, optical communications and light detection and ranging (LIDAR).

Fiber lasers based on Erbium doped fiber (EDF) have been widely investigated [1-3]. EDF exhibits efficient laser emission with excellent beam quality in single-mode laser systems. However, the low single-mode pump power provided by laser diode sources with core-pumping techniques, limits the 
output power of the laser emission when single-clad fibers are used [4,5]. The advent of double-clad fibers (DCF) allowed the development of reliable high-power fiber lasers with single-mode emission without the use of a single-mode pump source. By using cladding-pumping technique it is feasible to achieve power scaling of rare-earth doped fiber lasers with a multi-mode pump source [6]. Therefore, has been demonstrated that DCF-based lasers can provide emission with high efficiency, good beam quality and heat dissipation at high output power performance [7-9]. The aforementioned advantages make DCF-based cladding pumped fiber lasers attractive to replace bulk solid-state lasers for high power applications. Particularly, Er/Yb DCFs (EYDCF) have been attractive for high power fiber lasers operating in the $1.55 \mu \mathrm{m}$ wavelength range $[4,5,7,10]$. In a EYDCF-based laser, the pumping absorption of erbium ions is significantly improved due to the wide cross section area of ytterbium ions and non-radiative energy transference between erbium and ytterbium ions [11]. With the use of an EYDCF as gain medium, the erbium broad emission bandwidth can provide high gain over an extended wavelength range in the $1.55 \mu \mathrm{m}$ region which makes EYDCF-based fiber lasers attractive for the design of high-power tunable sources.

In EDF lasers at low pump power, the complete population inversion is not maintained over the entire length of the fiber. Then, the gain medium acts as a saturable absorber. The EYDCF core is highly co-doped with ytterbium to improve pump absorption and reduce ion pairs formation but not eliminated [10]. The erbium ion pairs excitation causes a ground state depletion resulting in laser pulsations at relaxation frequency of population inversion. The increase of pump power level replenishes the population inversion at a faster rate, then, the frequency of the SQS pulses is increased. This pulsing has typical shapes and characteristics of passive Q-switched pulses. Therefore, the technique to obtain these pulses is usually called self-Q-switching (SQS). Different approaches of SQS fiber laser have been reported [12-14].

On the other hand, efficient operation of tunable fiber lasers can be achieved by using fiber Bragg gratings (FBG) as wavelength spectral filters. The central wavelength of the FBG can be displaced by temperature or mechanical strain application $[15,16]$. Then, FBGs are reliable devices for wavelength tuning of the generated laser lines of fiber lasers [17-19].

Continuous wave $(\mathrm{CW})$ tunable dual-wavelength fiber lasers with high-efficiency have been of persistent interest for design of pump sources for $2 \mu \mathrm{m}$ laser emission, nonlinear effect research, free-space instrumentation and sensing, LIDAR and terahertz generation. However, rare-earth doped fibers are homogeneous gain media at room temperature resulting in a strong competition between the two generated laser lines that leads to unstable laser generation. Stable dual-wavelength laser emission can be obtained through an adjustment of intra-cavity losses. From previous investigations regarding dual-wavelength fiber lasers, the use of a fiber optical loop mirror (FOLM) with a high-birefringence (Hi-Bi) fiber loop has been theoretically and experimentally demonstrated as a trustworthy method for intra-cavity losses adjustment. The method is based in the wavelength displacement of the Hi-Bi FOLM periodical spectrum by applying temperature variations on the Hi-Bi fiber loop to finely balance the losses at the two simultaneously generated wavelengths [20]. In this regard, a stable high-efficiency dual-wavelength fiber laser operating in the $1.55 \mu \mathrm{m}$ waveband can be an attractive light source for different research areas.

In this paper, we report a high-efficiency dual-wavelength laser emission of a linear cavity EYDCF laser with SQS and CW operation depending on the pump power range. SQS operation is observed with low pump power in a range from 322 to $890 \mathrm{~mW}$. Pulses with minimum pulse duration of $\sim 1.5 \mu \mathrm{s}$ and maximum pulse energy of $\sim 3.5 \mu \mathrm{J}$ are obtained with the maximal pump power for SQS regime. With pump power up to $890 \mathrm{~mW}$, stable CW laser operation is achieved. Tunable dual-wavelength laser emission with maximum separation of $7 \mathrm{~nm}$ is obtained by using a pair of tunable FBGs for laser wavelengths selection. The intra-cavity losses adjustment to achieve dual-wavelength laser emission with equal powers is obtained by applying temperature variations on the fiber loop of the Hi-Bi FOLM. Slope efficiency of $36.3 \%$ and maximal output power of $3.6 \mathrm{~W}$ with dual-wavelength laser lines spectral linewidth of $\sim 0.2 \mathrm{~nm}$ is experimentally obtained. 


\section{Experimental Setup}

Figure 1 shows the proposed fiber laser experimental setup. The linear cavity is formed by two cascaded tunable fiber Bragg gratings (TFBG) in one side and a Hi-Bi FOLM in the opposite side. The gain medium is an EYDCF (Coractive DCF-EY-10/128) (Coractive High-Tech Inc., Quebec, QC, Canada) with length of $3.3 \mathrm{~m}$. The EYDCF is pumped at $976 \mathrm{~nm}$ by a multimode high-power laser source (Focuslight Technologies Inc., Xi'an, China) through a beam combiner. A 90/10 coupler is used as the laser power output, measured at the $90 \%$ output coupler port (output 1). An optical isolator (ISO) is used to protect the pump source of back light emission. The Hi-Bi FOLM consists of a 50/50 coupler forming a loop with output ports interconnected by a Hi-Bi fiber with length of $\sim 52 \mathrm{~cm}$ and birefringence of $4.125 \times 10^{-4}$. The Hi-Bi FOLM whose exhibits a wavelength-dependent periodical transmission spectrum is used as broad bandwidth wavelength reflector. The splices between the Hi-Bi fiber and the 50/50 coupler output ports were placed in mechanical rotation stages to adjust the fringe contrast of the reflection/transmission output spectrum [18]. The Hi-Bi fiber was placed on a Peltier device in order to perform a wavelength displacement of the Hi-Bi FOLM output spectrum by temperature variation applied on the Hi-Bi fiber loop [17-19]. The input port (output 2) is used to measure the Hi-Bi FOLM transmission spectrum and the laser spectra with an optical spectrum analyzer (OSA, Yokogawa AQ6375) (Yokogawa Corporation of America, Newnan, GA, USA) with spectral resolution of $0.03 \mathrm{~nm}$. The output 2 is also used to detect SQS pulses by a photodetector (Thorlabs, Newton, NJ, USA) and observed on an oscilloscope (Tektronix, Beaverton, OR, USA). TFBG1 and TFBG2, used as narrow bandwidth wavelength reflectors, exhibit $\sim 99 \%$ of maximum reflection at central reflection wavelengths of $1548.6 \mathrm{~nm}$ and $1543.86 \mathrm{~nm}$, respectively. The TFBGs were placed in a micrometric screw mechanical system for wavelength tuning by mechanical strain application [17-19].

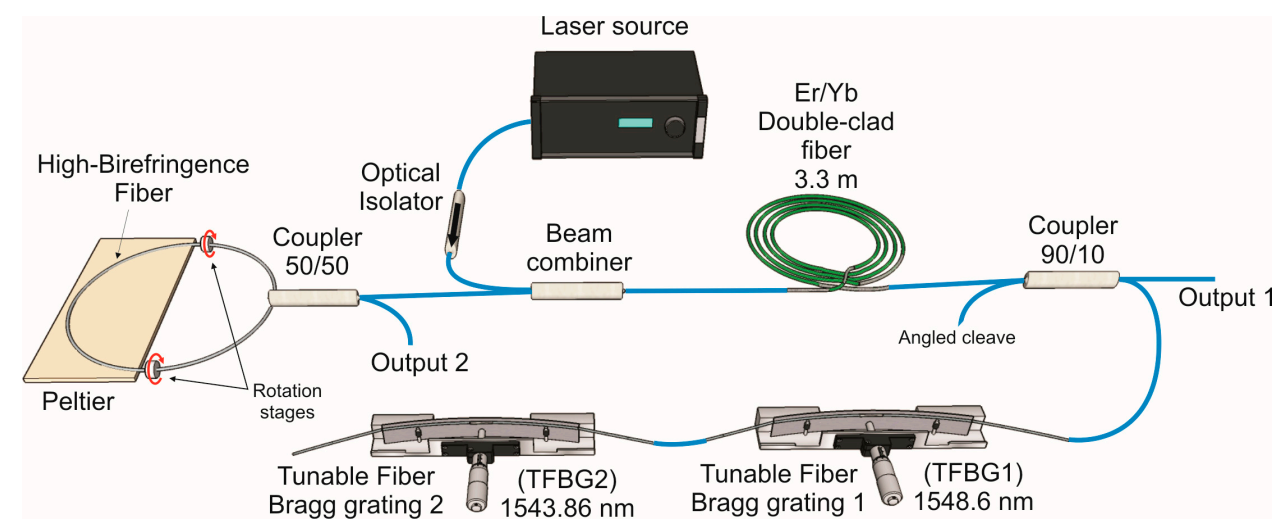

Figure 1. Experimental setup of the tunable dual-wavelength $\mathrm{Er} / \mathrm{Yb}$ double-clad fiber (EYDCF) laser.

\section{Experimental Results and Discussion}

The Hi-Bi FOLM output reflection/transmission exhibits sinusoidal spectrum which depends of the input wavelength. With the Hi-Bi fiber characteristics, the calculated wavelength period is $11.2 \mathrm{~nm}$ [20]. Figure 2 shows the Hi-Bi FOLM transmission spectrum measured using the EYDCF amplified spontaneous emission (ASE) input signal at the pump power below the lasing threshold of $322 \mathrm{~mW}$. The measurements were obtained at the Output 2 with the OSA. The Hi-Bi FOLM transmission spectrum was obtained in a wavelength range from 1540 to $1570 \mathrm{~nm}$ at $22.87^{\circ} \mathrm{C}$ of Hi-Bi fiber loop temperature. By adjusting the rotation stages, the Hi-Bi FOLM was set for maximum contrast between maximum and minimum transmission. As it can be observed, the measured Hi-Bi FOLM transmission spectrum wavelength period is of $\sim 11.13 \mathrm{~nm}$ for the wavelength period in which laser emission is expected because of the TFBGs central wavelengths. The output signal ratio between the Hi-Bi FOLM reflection values at the two TFBG wavelengths varies with the tuned wavelengths by the TFBGs. Then, a proper wavelength displacement of the Hi-Bi FOLM spectrum by Hi-Bi fiber loop temperature 
variations is performed to balance the internal cavity losses in order to obtain dual wavelength laser operation with equal powers.

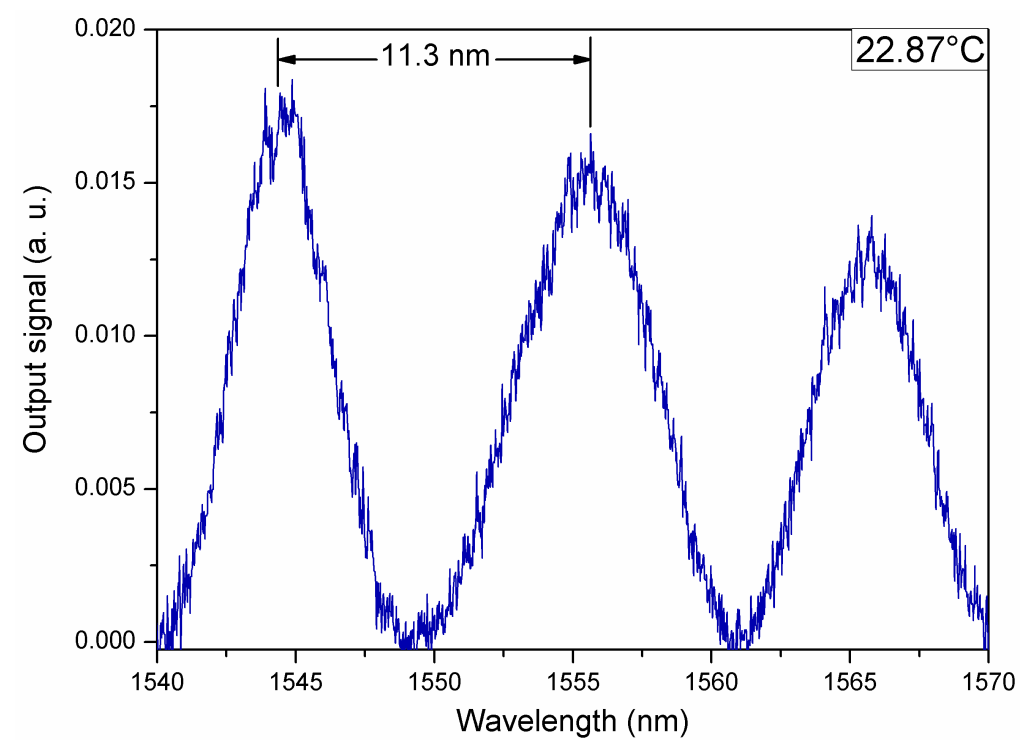

Figure 2. The transmission spectrum of the Hi-Bi fiber optical loop mirror (FOLM) output signal.

Figure 3a shows the measured output spectra of dual-wavelength fiber laser operation for different separations of the generated laser wavelengths. The measurements were obtained at the output 2 with the OSA with pump power of $2 \mathrm{~W}$. As it can be observed, dual-wavelength laser operation is achieved with maximum wavelengths separation of $7 \mathrm{~nm}$. Initially, TFBG1 and TFBG2 were tuned at 1546 and $1547 \mathrm{~nm}$, respectively, in order to obtain a separation between the generated laser lines of $1 \mathrm{~nm}$. Wavelength tuning interval of $0.5 \mathrm{~nm}$ s applied to both TFBGs (toward longer wavelengths for FBG1 and toward shorter wavelengths for FBG2) each measurement in order to increase the wavelengths separation for $1 \mathrm{~nm}$ until reaching a maximal separation of $7 \mathrm{~nm}$. The full width at half maximum (FWHM) spectral bandwidth of the generated laser lines is of $\sim 0.2 \mathrm{~nm}$. The temperature of the Hi-Bi fiber loop required for dual-wavelength operation with equal output powers, adjusted for each wavelength tuning, is also shown. Figure $3 b$ shows the output spectrum for dual-wavelength laser generation with equal pump powers in logarithmic scale. The measurement was obtained for the case of wavelengths separation of $5 \mathrm{~nm}$ were the required Hi-Bi fiber temperature for the intra-cavity losses adjustment is of $21.56{ }^{\circ} \mathrm{C}$. As it can be observed, the amplitude of both generated laser lines is determined by the Hi-Bi FOLM transmission spectrum gain. The 3-dB linewidth of both laser lines is of $\sim 0.19 \mathrm{~nm}$. Dual-wavelength operation with equal output powers is achieved by adjusting the intra-cavity losses for both generated laser lines with temperature variation on the FOLM Hi-Bi fiber loop for each wavelength tuning.

Figure 4 shows the FOLM Hi-Bi loop temperature adjustment required to achieve dual wavelength operation with equal output powers for different wavelengths separations $(\Delta \lambda)$ from experimental results obtained in Figure 3. As it is shown, dual-wavelength laser operation with equal output powers is obtained at temperatures of 23.50 and $21.56{ }^{\circ} \mathrm{C}$ of the Hi-Bi fiber loop for the minimal separation of 1 $\mathrm{nm}$ and the maximal separation of $7 \mathrm{~nm}$, respectively. As it can be observed, the Hi-Bi loop temperature required to obtain dual-wavelength operation decreases with the increase of the tuned wavelengths separation. By decreasing the temperature of the Hi-Bi fiber loop, the modulation spectrum of the FOLM shifts toward longer wavelengths. Then, the obtained results show a dual-wavelength laser emission with preference to generate the shorter laser wavelength which is balanced with a wavelength shift of the FOLM spectrum toward longer wavelength by decreasing the temperature of the Hi-Bi fiber loop [20]. As it is shown, the intra-cavity losses adjustment is more pronounced for 
shorter wavelengths separations of the generated laser lines. The intra-cavity losses adjustment is less significant for wavelengths separations of 5, 6 and $7 \mathrm{~nm}$.

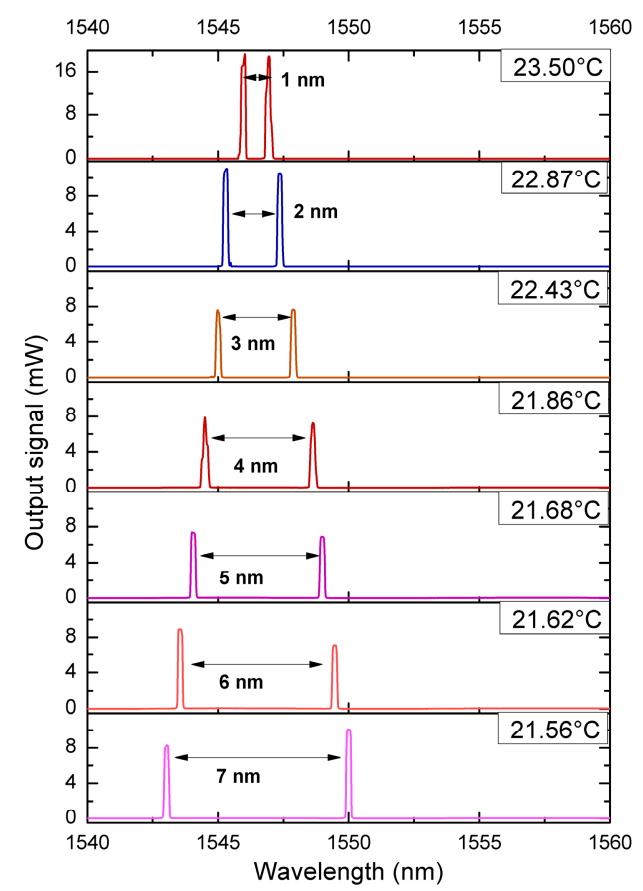

(a)

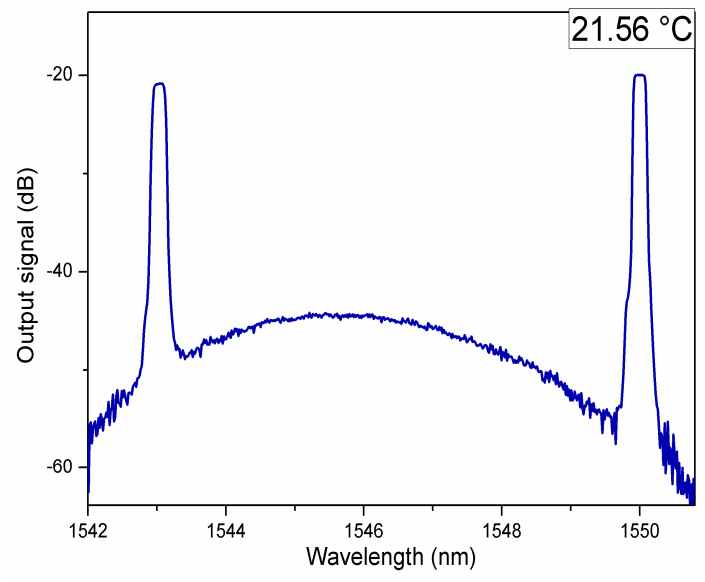

(b)

Figure 3. Continuous wave (CW) operation of the laser: (a) dual-wavelength laser emission with equal output powers for different wavelengths separations; (b) cavity losses adjustment by Hi-Bi fiber loop temperature for dual-wavelength laser generation with equal output powers and separation of $7 \mathrm{~nm}$.

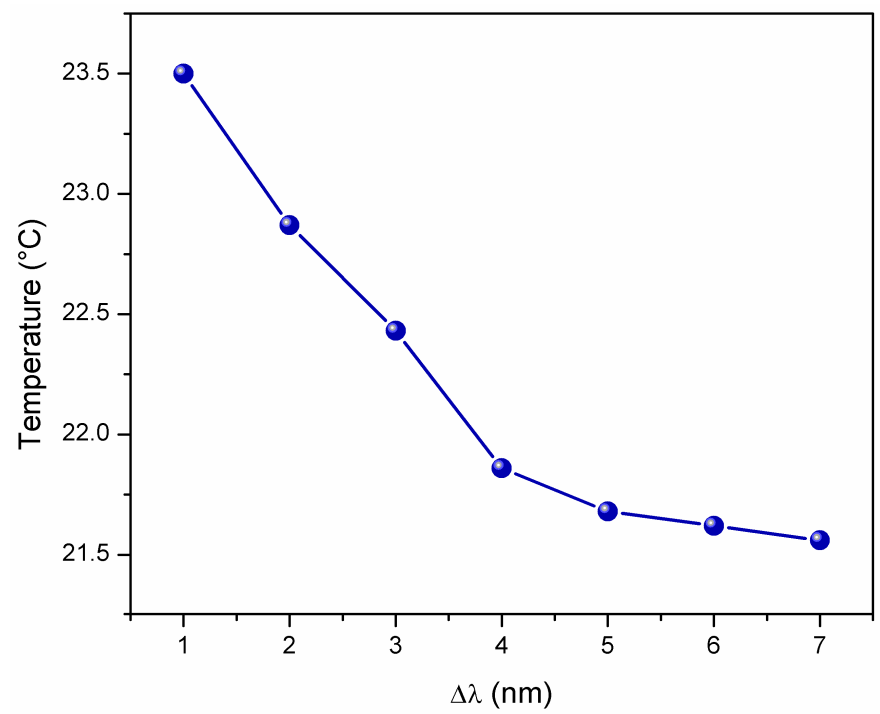

Figure 4. The temperature dependence of the Hi-Bi fiber loop on the wavelength separation of the generated laser lines to obtain dual-wavelength laser emission with equal output powers.

Figure 5 shows the EYDCF laser output power for dual-wavelength emission with equal output powers as a function of the applied pump power. The output power measurements were obtained at the output 1 with a power meter. The wavelength separation was tuned to $5 \mathrm{~nm}$ with a Hi-Bi loop temperature of $21.68{ }^{\circ} \mathrm{C}$ with simultaneous laser emission with equal output powers at 1544 and 
$1549 \mathrm{~nm}$. Initially, the pump power was set to $322 \mathrm{~mW}$, slightly above where laser emission is reached and the Hi-Bi fiber loop temperature was adjusted for dual-wavelength laser operation with equal output powers. With the pump power variation, equal output powers of the two simultaneously generated laser lines remain stable. Therefore, a Hi-Bi loop temperature change for intra-cavity losses adjustment as the pump power is varied is not required. The laser output power as a function of the pump power can be linearly fitted with a slope efficiency of $36.4 \%$. With the decrease of the separation between the generated laser lines, the efficiency increases because of the EYDCF ASE gain profile. For the maximal pump power applied of $10 \mathrm{~W}$ (limited to avoid damage of the cavity elements), the output power was of $3.6 \mathrm{~W}$. As it can be observed, two different operation regions are indicated. From $322 \mathrm{~mW}$ (the minimal pump power in which laser emission is reached) to $890 \mathrm{~mW}$, the EYDCF laser exhibits SQS laser operation. Then, CW laser operation is reached from the maximum limit for SQS operation.

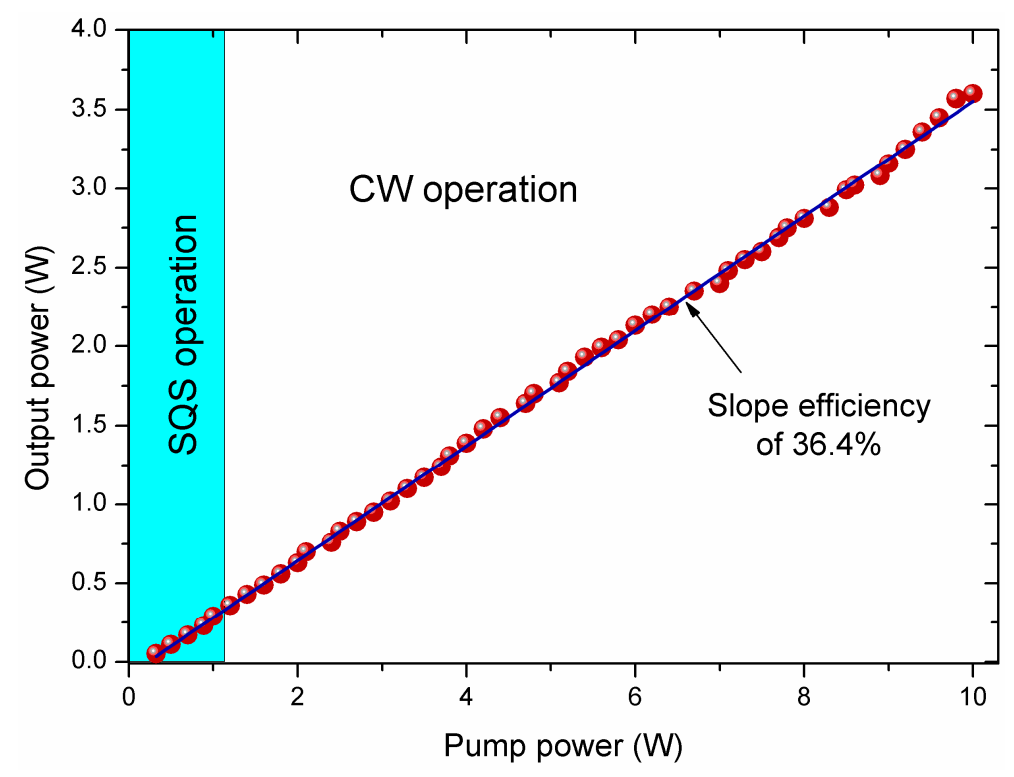

Figure 5. The laser output power for dual-wavelength emission as a function of the pump power (Self Q-switched (SQS) and CW laser operation regions are also indicated).

Figure 6 shows the SQS pulses trains evolution as a function of the pump power. The experimental results were performed with the required settings for the dual-wavelength laser operation presented in Figure 5. The measurements were obtained over a pump power range from $322 \mathrm{~mW}$ to $1 \mathrm{~W}$. The pulses were detected with a photo-detector and observed with an oscilloscope at the output 2. In the SQS operation region of the laser, when low pump power level is applied, the weakly pumped section of the EYDCF is acting as saturable absorber due to the formation of erbium ion pairs (on highly doped fibers, which is the case), regardless of the EYDCF length. With the increase of the pump power, the population inversion is replenished to a faster rate contributing to generate high energy pulses at increased frequency due to the amplification of the relaxation oscillations at lower pump level, depending on the EYDCF length [21]. Therefore, laser pulse trains with typical characteristics of passive Q-switched pulses were detected in a range from 322 to $890 \mathrm{~mW}$ in which SQS laser operation is obtained. As it can be observed, the repetition rate of the SQS pulses increases as the pump power is increased. The obtained experimental results were used to define the laser operation regions shown in Figure 5. With a pump power of $1.1 \mathrm{~W}, \mathrm{CW}$ laser operation is obtained when the gain along the EYDCF is more uniform reaching a level in which the effects of saturable absorption and relaxation oscillations are eliminated [21]. 


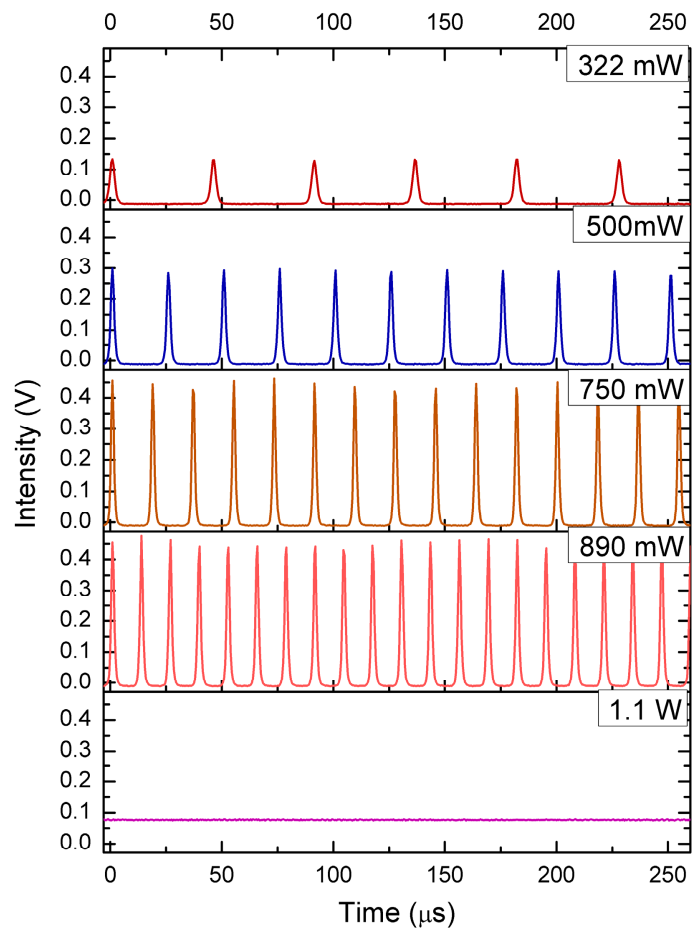

Figure 6. The pulses trains of the SQS laser operation as a function of the pump power for dual-wavelength laser emission.

Figure 7 shows experimental results of the SQS laser operation as a function of the pump power. The measurements were obtained in the laser SQS region for dual wavelength laser operation with pump power variations in a range from 322 to $890 \mathrm{~mW}$. The dual-wavelength laser generation was adjusted for the settings of the results obtained in Figure 5. Figure 7a shows the SQS pulses repetition rate and the laser average power as a function of the pump power. In the SQS laser operation range, an increase of the repetition rate and the average power is observed when the pump power is increased. Maximal repetition rate of $71.8 \mathrm{kHz}$ and average power of $249 \mathrm{~mW}$ are obtained with the maximum pump power where SQS pulses are observed of $890 \mathrm{~mW}$. With the increase of the pump power, the repetition rate of SQS pulses increases to a limit in which the gain along the EYDCF is more uniform leading to a reduction of the saturable absorption and suppressing SQS operation at high pump. As a result, when a passive fiber with enough length to increase the photon lifetime in the resonator is not added, the full repetition rate of the SQS operation of the laser is mainly determined by the physical length of the EYDCF [21]. SQS laser pulses are observed in a range from 21.7 to $71.63 \mathrm{kHz}$ for a pump range from 322 to $980 \mathrm{~mW}$. Figure $7 \mathrm{~b}$ shows the estimated pulse energy and the measured pulse duration as a function of the pump power applied. As it can be observed, the obtained pulses exhibit a typical behavior of passive Q-switched pulses. When the applied pump power increases, the pulse energy increases and the pulse duration decreases. With the maximal pump power of $890 \mathrm{~mW}$, the maximal pulse energy of $3.49 \mu \mathrm{J}$ and the minimal pulse duration of $1.5 \mu$ s were obtained. Figure 7c shows the estimated peak power of the SQS pulses as a function of the pump power. As it can be observed, the peak power increases as the pump power is increased. It is worth to note that high peak power pulses of around $2.3 \mathrm{~W}$ are obtained in the pump power limit between SQS and CW operations of the EYDCF laser. 


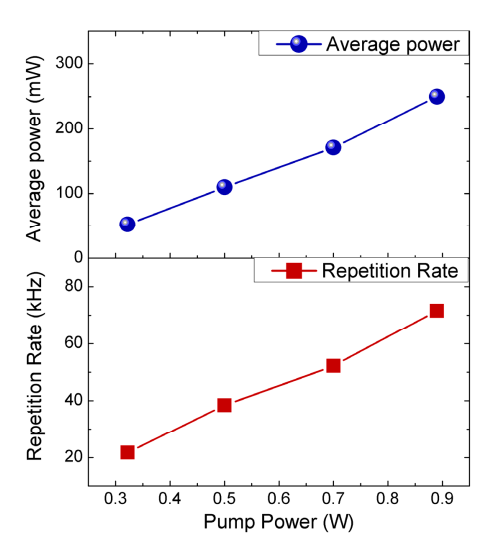

(a)

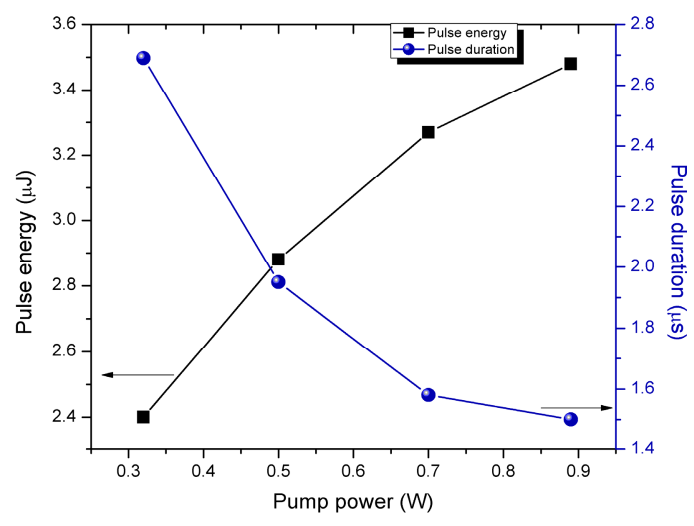

(b)

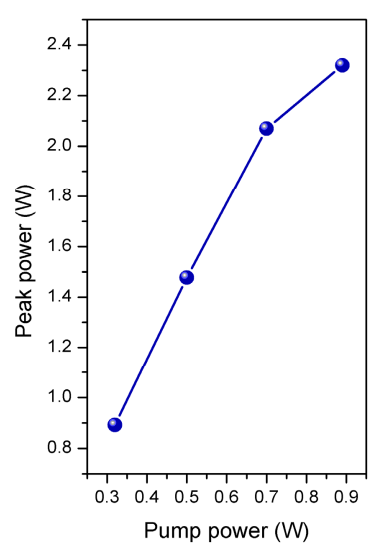

(c)

Figure 7. Characterization of the SQS laser pulses as a function of the pump power: (a) measured average power and repetition rate; (b) estimated pulse energy and measured pulse duration; (c) estimated pulses peak power.

\section{Discussion}

High-efficiency of all-fiber lasers can be reached by using FBGs for the selection and tuning of the generated laser lines in single laser emission. However, in order to obtain equal output powers of the simultaneously generated laser lines in dual-wavelength laser generation, the use of a method to balance the intra-cavity losses is required. In this regard, from the experimental results the Hi-Bi FOLM has been demonstrated as a reliable optical device for the fine intra-cavity losses adjustment. The wavelength displacement of the Hi-Bi FOLM by temperature variations on the Hi-Bi fiber stands for a straightforward and non-arbitrary method for the gain balancing of both generated laser lines in dual-wavelength fiber lasers instead of polarization controllers which are commonly used for this purpose.

On the other hand, from the obtained results it can be noticed that DCFs allow the increase the output power in the development of single-mode fiber lasers by using cladding-pumping technique with a multi-mode pump source. The use of DCFs in fiber lasers is an attractive option for replacement of bulk solid-state lasers in order to reach high power laser emission. However, in case of all-fiber lasers design, the high power provided is frequently restricted to the optical power handled by the fiber elements which form the cavity. Moreover, although the high ytterbium co-doping in EYDCF reduces the erbium ion pairs formation, the phenomenon occurs. As a consequence, SQS pulses are present in EYDCF fiber lasers. Regardless of the EYDCF length, under low pump power conditions the self-pulsations are determined by the relaxations oscillations. In addition, the EYDCF acts as saturable absorber due to the weakly pumped section of the EYDCF. When pump power is increased, the relaxation oscillations are amplified to a faster rate leading to the increase of pulses repetition rate, which is typical characteristic of passively Q-switched lasers. As the pump power is increased, the saturable absorption is reduced due to the increase of gain uniformity along the EYDCF, reaching a limit in which CW laser operation is obtained. As a consequence, in case of the proposed configuration when pump power is increased, the full repetition of the SQS laser operation depends on the EYDCF length [21]. In order to avoid possible damages in fiber systems caused by high energy SQS pulses generated at low pump power when a CW laser source is required or otherwise to establish possible applications of the SQS laser source with a wide repetition rate, the existence of SQS pulses in double-clad-fiber-based lasers has to be studied establishing operation limits, the conditions which contribute to their generation and the mechanisms to suppress or increase their operation according with the laser application. 


\section{Conclusions}

In this paper, SQS and CW operation of a linear cavity tunable dual-wavelength high-efficiency fiber laser based in EYDCF has been experimentally demonstrated. The SQS pulses are observed with low pump power application in a range from 322 to $890 \mathrm{~mW}$. The observed SQS pulses exhibit minimum pulse duration of $\sim 1.5 \mu$ s and maximum pulse energy of $\sim 3.5 \mu \mathrm{J}$ with the maximal pump power for SQS operation of $890 \mathrm{~mW}$. CW dual wavelength laser operation is achieved with application of pump power bellow of $890 \mathrm{~mW}$. Simultaneously generated laser lines with different wavelength tuned separations were obtained in a range from 1 to $7 \mathrm{~nm}$. A Hi-Bi FOLM was used to balance the intra-cavity losses to obtain dual wavelength laser operation with equal powers by Hi-Bi loop temperature variations in a range from 23.5 to $21.56^{\circ} \mathrm{C}$ for tuned wavelength separations from 1 to $7 \mathrm{~nm}$, respectively. A high-efficiency laser emission of $\sim 36.4 \%$ with maximal output power of $3.6 \mathrm{~W}$ with a pump power of $10 \mathrm{~W}$ was achieved.

Acknowledgments: This work was funded by CONACyT grant CB-256401. M. Durán-Sánchez and R. I. Álvarez-Tamayo want to thank the Cátedras CONACyT program.

Author Contributions: Manuel Durán-Sánchez and Ricardo I. Alvarez-Tamayo conceived and designed the experiments; Berenice Posada-Ramírez performed the experiments; Manuel Durán-Sánchez, Ricardo I. Alvarez-Tamayo, analyzed the data; Baldemar Ibarra-Escamilla and Evgeny A. Kuzin contributed reagents/materials/analysis tools; Ricardo I. Alvarez-Tamayo wrote the paper; Baldemar Ibarra-Escamilla, Evgeny A. Kuzin, Manuel Durán-Sánchez and Patricia Prieto-Cortés contributed to the manuscript writing revision and comments.

Conflicts of Interest: The authors declare no conflict of interest.

\section{References}

1. Chen, D. Stable multi-wavelength erbium-doped fiber laser based on a photonic crystal fiber Sagnac loop filter. Laser Phys. Lett. 2007, 4, 437-439. [CrossRef]

2. Liu, Z.Y.; Liu, Y.G.; Du, J.B.; Kai, G.Y.; Dong, X.Y. Tunable multiwavelength erbium-doped fiber laser with a polarization-maintaining photonic crystal fiber Sagnac loop filter. Laser Phys. Lett. 2008, 5, 446-448. [CrossRef]

3. Chen, D.; Fu, H.; Liu, W. Single-longitudinal-mode erbium-doped fiber laser based on a fiber Bragg grating Fabry-Perot filter. Laser Phys. 2007, 17, 1246-1248. [CrossRef]

4. Nilsson, J.; Alam, S.; Alvarez-Chavez, J.A.; Turner, P.W.; Clarkson, W.A.; Grudinin, A.B. High-power and tunable operation of erbium-ytterbium co-doped cladding-pumped fiber lasers. IEEE J. Quantum Electron. 2003, 39, 987-994. [CrossRef]

5. Sobon, G.; Kaczmarek, P.; Abramski, K.M. Erbium-ytterbium co-doped fiber amplifier operating at $1550 \mathrm{~nm}$ with stimulated lasing at $1064 \mathrm{~nm}$. Opt. Commun. 2012, 285, 1929-1933. [CrossRef]

6. Snitzer, E.; Po, H.; Hakimi, F.; Tumminelli, R.; McCollum, B.C. Double-clad offset core Nd fiber laser. Post-Deadline Paper PD5. In Proceedings of the Optical Fiber Sensors OFS'88, New Orleans, LA, USA, 27 January 1988. [CrossRef]

7. González-García, A.; Ibarra-Escamilla, B.; Pottiez, O.; Kuzin, E.A.; Maya-Ordoñez, F.; Duran-Sánchez, M.; Deng, C.; Haus, J.W.; Powers, P.E. High efficiency, actively Q-switched Er/Yb fiber laser. Opt. Laser Technol. 2013, 48, 182-186. [CrossRef]

8. Liu, C.; Ye, C.; Luo, Z.; Cheng, H.; Wu, D.; Zheng, Y.; Liu, Z.; Qu, B. High-energy passively Q-switched 2 $\mu \mathrm{m} \mathrm{Tm}{ }^{3+}$-doped double-clad fiber laser using graphene-oxide-deposited fiber taper. Opt. Express 2013, 21, 204-209. [CrossRef] [PubMed]

9. Huang, J.Y.; Huang, S.C.; Chang, H.L.; Su, K.W.; Chen, Y.F.; Huang, K.F. Passive Q switching of Er-Yb fiber laser with semiconductor saturable absorber. Opt. Express 2008, 16, 3002-3007. [CrossRef] [PubMed]

10. Jeong, Y.; Yoo, S.; Codemard, C.A.; Nilsson, J.; Sahu, J.K.; Payne, D.N.; Horley, R.; Turner, P.W.; Hickey, L.; Harker, A.; et al. Erbium: Ytterbium Codoped Large-Core Fiber Laser With 297-W Continuous-Wave Output Power. IEEE J. Sel. Top. Quantum Electron. 2007, 13, 573-579. [CrossRef] 
11. Harun, S.W.; Abdul-Rashid, H.A.; Muhd-Yassin, S.Z.; Abd-Rahman, M.K.; Tamjis, M.R.; Ahmad, H. Dual-stage $\mathrm{Er} / \mathrm{Yb}$ doped fiber amplifier for gain and noise figure enhancements. IEICE Electron. Express 2006, 3, 517-521. [CrossRef]

12. Kir'yanov, A.V.; Il'ichev, N.N.; Barmenkov, Y.O. Excited-state absorption as a source of nonlinear thermo-induced lensing and self-Q-switching in an all-fiber Erbium laser. Laser Phys. Lett. 2004, 1, 194-198. [CrossRef]

13. Kir'yanov, A.V.; Barmenkov, Y.O. Self-Q-switched Ytterbium-doped all-fiber laser. Laser Phys. Lett. 2006, 3, 498-502. [CrossRef]

14. Álvarez-Tamayo, R.I.; Durán-Sánchez, M.; Pottiez, O.; Ibarra-Escamilla, B.; Bello-Jiménez, M.; Kuzin, E.A. Self-Q-switched Er-Yb double clad fiber laser with dual wavelength or tunable single wavelength operation by a Sagnac interferometer. Laser Phys. 2015, 25, 075102. [CrossRef]

15. Du, W.-C.; Tao, X.-M.; Tam, H.-Y. Fiber Bragg grating cavity sensor for simultaneous measurement of strain and temperature. IEEE Photon. Technol. Lett. 1999, 11, 105-107. [CrossRef]

16. Jung, J.; Nam, H.; Lee, J.H.; Park, N.; Lee, B. Simultaneous measurement of strain and temperature by use of a single-fiber Bragg grating and an erbium-doped fiber amplifier. Appl. Opt. 1999, 38, 2749-2751. [CrossRef] [PubMed]

17. Álvarez-Tamayo, R.I.; Durán-Sánchez, M.; Pottiez, O.; Kuzin, E.A.; Ibarra-Escamilla, B. Tunable dual-wavelength fiber laser based on a polarization-maintaining fiber Bragg grating and a Hi-Bi fiber optical loop mirror. Laser Phys. 2011, 21, 1932-1935. [CrossRef]

18. Durán-Sánchez, M.; Flores-Rosas, A.; Álvarez-Tamayo, R.I.; Kuzin, E.A.; Pottiez, O.; Bello-Jiménez, M.; Ibarra-Escamilla, B. Fine adjustment of cavity loss by Sagnac loop for a dual wavelength generation. Laser Phys. 2010, 20, 1270-1273. [CrossRef]

19. Álvarez-Tamayo, R.I.; Durán-Sánchez, M.; Pottiez, O.; Ibarra-Escamilla, B.; Cruz, J.L.; Andrés, M.V.; Kuzin, E.A. A dual-wavelength tunable laser with superimposed fiber Bragg gratings. Laser Phys. 2013, 23, 055104. [CrossRef]

20. Álvarez-Tamayo, R.I.; Durán-Sánchez, M.; Pottiez, O.; Kuzin, E.A.; Ibarra-Escamilla, B.; Flores-Rosas, A. Theoretical and experimental analysis of tunable Sagnac high-birefringence loop filter for dual-wavelength laser application. Appl. Opt. 2011, 50, 253-260. [CrossRef] [PubMed]

21. Upadhyaya, N.B.; Kuruvilla, A.; Chakravarty, U.; Shenoy, M.R.; Thyagarajan, K.; Oak, S.M. Effect of laser linewidth and fiber length on self-pulsing dynamics and output stabilization of single-mode $\mathrm{Yb}$-doped double-clad fiber laser. Appl. Opt. 2010, 49, 2316-2325. [CrossRef] [PubMed] 